\title{
Microlensing towards the Large Magellanic Cloud: a study of the LMC halo contribution
}

\author{
S. Calchi Novati ${ }^{1,2,4}$, F. De Luca ${ }^{1,2}$, Ph. Jetzer $^{1}$, and G. Scarpetta ${ }^{2,3,4}$ \\ ${ }^{1}$ Institute for Theoretical Physics, University of Zürich, Winterthurerstrasse 190, 8057 Zürich, Switzerland \\ e-mail: novati@physik.unizh.ch \\ 2 Dipartimento di Fisica "E. R. Caianiello", Università di Salerno, Via S. Allende, 84081 Baronissi (SA), Italy \\ 3 International Institute for Advanced Scientific Studies, Vietri sul Mare (SA), Italy \\ ${ }^{4}$ Istituto Nazionale di Fisica Nucleare, sez. Napoli, Italy
}

Received 7 March 2006 / Accepted 10 July 2006

ABSTRACT

\begin{abstract}
We carry out a new analysis of the sample of MACHO microlensing candidates towards the LMC. Our main purpose is to determine the lens population to which the events may belong. We give particular emphasis to the possibility of characterising the Milky Way dark matter halo population with respect to the LMC one. Indeed, we show that only a fraction of the events have characteristics that match those expected for lenses belonging to the MACHO population of the Galactic halo. This suggests that this component cannot explain all the candidates. Accordingly, we challenge the view that the dark matter halo fraction of both the Galaxy and the LMC halos are equal, and indeed we show that, for a MACHO mass in the range $0.1-0.3 M_{\odot}$, the LMC halo fraction can be significantly larger than the Milky Way's. In this perspective, our main conclusion is that up to about half of the observed events could be attributed to the LMC MACHO dark matter halo.
\end{abstract}

Key words. gravitational lensing - Galaxy: halo - galaxies: Magellanic clouds - cosmology: dark matter

\section{Introduction}

Since the original proposal of Paczyński (1986), microlensing has been shown to be a powerful tool for investigating the MACHO composition of galactic halos. The microlensing surveys towards the LMC and the SMC probed the existence of compact halo objects along these lines of sight; however, the assessment of these results with respect to the actual fraction of the Milky Way (hereafter "MW") halo in the form of MACHOs is still highly debated.

The MACHO collaboration reported the detection of 13-17 microlensing candidates towards the LMC (Alcock et al. 2000b), arguing in favour of a MACHO halo fraction of $~ 20 \%$ of objects of $\sim 0.5 M_{\odot}$ and estimating a microlensing optical depth towards the LMC of $\tau=1.2_{-0.3}^{+0.4} \times 10^{-7}$. The reported microlensing rate towards the LMC significantly exceeds the expected one from known visible components of our Galaxy. Further analysis mainly confirmed these conclusions (Bennett et al. 2005). On the other hand, the EROS collaboration, from observations towards both the LMC and the SMC, put more and more lower upper limits on the MACHO contribution to galactic halos (Lasserre et al. 2000; Afonso et al. 2003; Tisserand \& Milsztajn 2005), which are no longer compatible with the MACHO results.

These questions have also been addressed by microlensing surveys towards M 31 (Ansari et al. 1997), and a first evidence of a MACHO contribution along this line of sight have been reported (Calchi Novati et al. 2005), although challenged in de Jong et al. (2006). Overall, the picture remains unclear, in particular the problem of the nature and location of the observed events. For example, Green \& Jedamzik (2002) have cast doubts on the interpretation of the microlensing data towards LMC as due to a dominant lens population made by Galactic halo MACHOs. In fact they show, at a high level of confidence, that the distribution of the duration of the observed microlensing events is significantly narrower than what is expected from a standard halo-lens population.

We have already addressed some of these questions in Jetzer et al. (2002) and Mancini et al. (2004) (hereafter Papers I and II, respectively). A main issue in both works is that the microlensing events towards the LMC, as observed by the MACHO collaboration, do not necessarily all belong to the same lens population. In particular in Paper II, we considered the issue of self lensing in the framework of the van der Marel et al. (2002) picture of the luminous components of the LMC. The main conclusion, based both on the predicted number and characteristics of self-lensing events, was that self lensing alone cannot explain all the observed events.

In the present work we extend the analysis by fully considering the LMC and MW dark matter halo MACHO lens populations. The main question we want to address is whether and to what extent events due to the lens population residing in the LMC halo can contribute to the observed rates. This issue was first proposed and discussed in Gould (1993). Here we consider again the question taking into account both the results of the $\mathrm{MACHO}$ collaboration and the most recent results on the modeling of the LMC.

The paper is organised as follows. In Sect. 2 we review the models of the LMC and Galactic components we consider. In Sect. 3 we discuss the evaluation of the microlensing rate and present the results for the expected number and duration of the microlensing events. In Sect. 4 we carry out our new analysis and present our main conclusions on the LMC MACHO halo contribution, and in Sect. 5 we present our conclusions. 


\section{Models}

We consider the LMC as composed of a luminous part, the bar, and the disc, plus a stellar and a dark matter halos. We also include the Galactic dark matter halo, but we do not include the Galactic disc and spheroid populations. These components have already been shown to give smaller contributions than the LMC self lensing (Alcock et al. 2000b), which we include instead. Accordingly, we exclude from our analysis the single event whose lens, upon a direct search, has been acknowledged to be part of these components.

For the structure and dynamics of the luminous components of the LMC we follow Paper II closely. From the analysis in van der Marel et al. (2002), who derive their results on the assumption that the carbon star population is representative of the bulk of the LMC disc stars, we take up the results on the LMC disc intrinsic ellipticity, vertical thickness, lineof-sight velocity dispersion, rotation curve, and the coincidence of the centre of mass of the disc and the bar components. We assume the following values for the bar and disc components $M_{\mathrm{bar}}+M_{\mathrm{disc}}=(2.7 \pm 0.6) \times 10^{9} M_{\odot}($ van der Marel et al. 2002), and $M_{\text {bar }}=1 / 5 M_{\text {disc }}$ (Gyuk et al. 2000), which we consider to be both centred at $\alpha, \delta=5^{\mathrm{h}} 27.6^{\mathrm{m}} \pm 3.9^{\mathrm{m}},-69.87^{\circ} \pm 0.41^{\circ}(\mathrm{J} 2000)$ at a distance from us of $D_{0}=50.1 \pm 2.5 \mathrm{kpc}$ (van der Marel et al. 2002). We use the same density star distribution as in Paper II, characterised by a vertical distribution for the exponential disc described by a sech ${ }^{2}$ function and a boxy bar, with a Gaussian profile along the major axis and the section described by a $\exp \left(-r^{4}\right)$ function. The vertical structure of the LMC has been recently the object of an intensive debate (for a discussion see van der Marel 2004). We considered this issue with respect to the expected self lensing signal in Paper II. In the present analysis, where we focus on the contribution of the two halos, we do not enter into this discussion but consider only the configuration with coplanar disc and bar.

The presence of a significant LMC stellar halo population is a matter of debate (Minniti et al. 2003; Alves 2004; Gallart et al. 2004). In the present analysis we include the contribution of such a possible component following Alves (2004), who proposes a spherically symmetric spheroid with density profile

$\rho=\rho_{0}\left(1+\frac{R^{2}}{a_{\mathrm{C}}^{2}}\right)^{-k}$,

with $k=3 / 2$, central density $\rho_{0}=6.3 \times 10^{6} M_{\odot} \mathrm{kpc}^{-3}$, and core radius $a_{\mathrm{C}}=1.42 \mathrm{kpc}$ for a total mass, within $8.9 \mathrm{kpc}$, of $0.35 \times 10^{9} M_{\odot}$ somewhat smaller than that of the bar. The optical depth profile of this component shows a near-far asymmetry due to the LMC disc inclination, whose overall shape recalls that of the optical depth profile of the LMC MACHO halo component (Paper II, Fig. 4), with a maximum value around $0.9 \times 10^{-8}$ reached in correspondence of the field MACHO 6.

Following van der Marel et al. (2002), who present observational evidence based on the rotation curves, we include a significant LMC dark matter halo component. We assume a total LMC mass of $8.7 \times 10^{9} M_{\odot}$ within $8.9 \mathrm{kpc}$ with a truncation radius of $15 \mathrm{kpc}$ (van der Marel et al. 2002), which we consider to also include the contribution from the stellar halo. We assume a spherical isothermal model ( $k=1$ in Eq. (1)) with a core radius of $a_{\mathrm{C}}=2 \mathrm{kpc}$ (Alcock et al. 2000b).

This spherical symmetric configuration might not be a realistic description of this component given that the dynamical environment of the LMC can induce tidal distortions and disruptions, especially in the outer parts. To take this issue into account, in
Paper II we compared the LMC halo optical depth profiles for both a spherical and an elliptical configuration (Figs. 4 and 5 respectively): the overall shape is similar even if in the latter case the maximum value rises by about $20 \%$ and the near-far asymmetry is enhanced. However, as we lack any strong constraint, we prefer not to introduce a further parameter in the present analysis; therefore, we only consider the spherical configuration, in view also of the possibility of carrying out a more direct comparison with previous works.

In this same perspective, we consider the "standard" isothermal profile for the Galactic dark matter halo with a core radius of $5 \mathrm{kpc}$, local density $7.9 \times 10^{6} M_{\odot} \mathrm{kpc}^{-3}$, and a distance from the Galactic centre of $8.5 \mathrm{kpc}$ (Alcock et al. 2000b).

\section{The microlensing rate}

The main tool of investigation we use is $\frac{\mathrm{d} \Gamma}{\mathrm{d} T_{\mathrm{E}}}$, the differential rate of microlensing events with respect to the Einstein time $T_{\mathrm{E}}$ (de Rujula et al. 1991; Griest 1991; Roulet \& Mollerach 1997). This allows us to make predictions on the timescale, the number, and the spatial distribution of the expected events, which we can compare with the corresponding observed quantities. With respect to the self-lensing configuration that we analysed in Paper II (Sect. 4.2), we can no longer adopt the useful approximation $\frac{D_{\mathrm{ol}}}{D \text { os }} \equiv x \approx 1$. Moreover we now have to take the bulk velocity of the LMC components and the relative motion between the LMC and the Galaxy (van der Marel et al. 2002) fully into account.

The source stars belong to the luminous components, disc or bar, of the LMC, whereas the lenses can belong either to the LMC or the MW halo. We assume an isotropic Maxwellian profile $^{1}$ for the velocity distribution for both lenses and sources. For the flattened LMC luminous components, this is a rough approximation; still, it gives a fair description of the average properties of these populations that we consider to be sufficient in the present framework.

For the LMC disc component we consider the rotational velocity as in van der Marel et al. (2002) with $\sigma=20.2 \mathrm{~km} \mathrm{~s}^{-1}$. For the LMC bar stars we use a higher value of the velocity dispersion than for the disc, $\sigma=24.7 \mathrm{~km} \mathrm{~s}^{-1}$ (Cole et al. 2005). For both the LMC halo components, stellar and MACHO, we use $\sigma=46 \mathrm{~km} \mathrm{~s}^{-1}$ (van der Marel et al. 2002; Alves 2004) (we tested that our results remain qualitatively unaltered by changing this value up to $20 \%$ ); for the Galactic halo, $\sigma=155 \mathrm{~km} \mathrm{~s}^{-1}$.

The expression for the random motion velocity for the lenses reads $^{2}$

$\boldsymbol{v}_{l \perp}=\hat{\boldsymbol{v}}_{l \perp}+x \boldsymbol{v}_{\mathrm{s} \perp}+\boldsymbol{A}_{\perp}$

where $\hat{\boldsymbol{v}}_{l \perp}$ is the velocity relative to the microlensing tube at position $x$, whose modulus is the ratio between the Einstein radius and the Einstein time $\left(\hat{v}_{l \perp}=R_{\mathrm{E}} / T_{\mathrm{E}}\right)$, and $\boldsymbol{v}_{\mathrm{S} \perp}$ the random component of the velocity of the sources. All the bulk motions are included in $\boldsymbol{A}_{\perp}$, defined as

$$
\begin{aligned}
\boldsymbol{A}_{\perp}= & \tilde{\boldsymbol{v}}_{\odot \perp}+x\left(\tilde{\boldsymbol{v}}_{\mathrm{LMC} \perp}-\tilde{\boldsymbol{v}}_{\odot \perp}+\boldsymbol{v}_{\mathrm{s}, \mathrm{drift} \perp}\right) \\
& -\eta\left(\tilde{\boldsymbol{v}}_{\mathrm{LMC} \perp}+\boldsymbol{v}_{l, \mathrm{drift} \perp}\right)
\end{aligned}
$$

1 The Maxwellian profile of the velocity distribution is the first term of a series expansion in terms of Gauss-Hermite moments (van der Marel \& Franx 1993; Gerhard 1993). See Sect. 3.2 of Paper I.

2 The velocity components parallel to the line of sight are integrated out, and the subscript $\perp$ indicates the vectorial component in the plane orthogonal to the line of sight. 


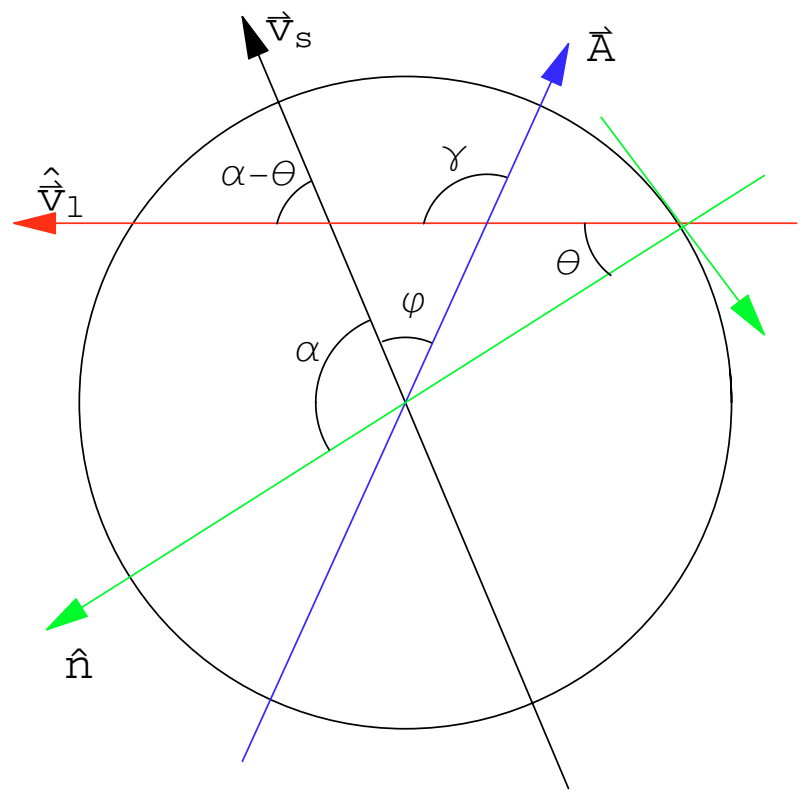

Fig. 1. Scheme of a section of the microlensing tube with indicated the positions of the vectors and the angles involved.

where $\eta=0,1$ for lenses in the Galaxy and in the LMC, respectively; $\boldsymbol{v}_{l, \text { drift } \perp}$ is the drift velocity of the lens star belonging to the LMC disc (for the bar sources as well as for the halo lenses we only consider a random motion component), and a tilde over a vector indicates a quantity measured by an observer comoving with the Galactic centre. In the self-lensing configuration, $\boldsymbol{A}_{\perp} \approx 0$, and Eq. (2) reduces to Eq. (13) of Paper II.

We call $\alpha$ the angle between the inner normal to the tube, $\hat{\boldsymbol{n}}$, and the source velocity $\boldsymbol{v}_{\mathrm{s} \perp} ; \theta$ the angle between $\hat{\boldsymbol{n}}$ and $\hat{\boldsymbol{v}}_{l \perp}$, $\theta \in(-\pi / 2, \pi / 2) ; \varphi$ the angle between $\boldsymbol{v}_{\mathrm{S} \perp}$ and $\boldsymbol{A}_{\perp}$; so that the angle between $\boldsymbol{v}_{l \perp}$ and $\boldsymbol{v}_{\mathrm{s} \perp}$ is $\alpha-\theta$, and that between $\boldsymbol{v}_{l \perp}$ and $\boldsymbol{A}_{\perp}$ is $\gamma=\alpha+\varphi-\theta$. Both $\alpha$ and $\varphi$ vary in the range $(0,2 \pi)$ (Fig. 1).

In the case of $\boldsymbol{A}_{\perp}=0$, all the integrations over the angular variables can be carried out analytically. This is now only possible for the $\theta$ variable. Altogether, after an analytical integration on the modulus of the source velocity, we are left with the following expression of the differential rate with respect to the Einstein time $T_{\mathrm{E}}$ :

$$
\begin{aligned}
\frac{\mathrm{d} \Gamma}{\mathrm{d} T_{\mathrm{E}}}= & \int_{0}^{2 \pi} \mathrm{d} \alpha \int_{0}^{2 \pi} \mathrm{d} \varphi \int_{d_{\min }}^{d_{\max }} \mathrm{d} D_{\mathrm{os}} \int_{x_{\min }}^{1} \mathrm{~d} x \int_{\mu_{\min }}^{\mu_{\max }} \mathrm{d} \mu \\
& \times \frac{\mathrm{d} n_{l}}{\mathrm{~d} \mu} \frac{D_{\mathrm{os}} \rho_{l} \rho_{\mathrm{s}} \hat{v}_{l \perp}^{4}}{2 \pi^{2}\left(\sigma_{l}^{2}+x^{2} \sigma_{\mathrm{s}}^{2}\right) \mathcal{N}} \\
& \times\left\{\exp \left[-\frac{A^{2}+\hat{v}_{l \perp}^{2}+2 A \hat{v}_{l \perp} \cos (\alpha+\varphi)}{2 \sigma_{l}^{2}}\right]\right. \\
& -\frac{\sqrt{\pi} x \sigma_{\mathrm{s}}\left(\hat{v}_{l \perp} \cos (\alpha)+A \cos (\varphi)\right)}{\sigma_{l} \sqrt{2\left(\sigma_{l}^{2}+x^{2} \sigma_{\mathrm{s}}^{2}\right)}} \\
& \times \exp \left[-\frac{A^{2}+\hat{v}_{l \perp}^{2}+2 A \hat{v}_{l \perp} \cos (\alpha+\varphi)}{2\left(\sigma_{l}^{2}+x^{2} \sigma_{\mathrm{s}}^{2}\right)}\right. \\
& \left.-\frac{x^{2} \sigma_{\mathrm{s}}^{2}\left(\hat{v}_{l \perp} \sin (\alpha)-A \sin (\varphi)\right)^{2}}{\sigma_{l}^{2} 2\left(\sigma_{l}^{2}+x^{2} \sigma_{\mathrm{s}}^{2}\right)}\right] \\
& \times\left(1-\operatorname{Erf}\left(\frac{x \sigma_{\mathrm{s}}\left(\hat{v}_{l \perp} \cos (\alpha)+A \cos (\varphi)\right)}{\sigma_{l} \sqrt{2\left(\sigma_{l}^{2}+x^{2} \sigma_{\mathrm{s}}^{2}\right)}}\right)\right),
\end{aligned}
$$

where the normalization factor $\mathcal{N}$ is the integral over the line of sight of the sources

$\mathcal{N}=\int_{d_{\min }}^{d_{\max }} \mathrm{d} D_{\mathrm{os}} \rho_{\mathrm{s}}\left(D_{\mathrm{os}}\right)$,

having assumed that the number of detectable stars varies with the distance as $D_{\text {os }}^{-2}$. We define $x_{\text {min }}=d_{\min } / D_{\text {os }}, A=|\boldsymbol{A}|$; the integration limits along the line of sight, $d_{\min }$ and $d_{\max }$, represent the distances from the observer to the intersection with the LMC tidal surface, for lenses in the LMC, whereas $d_{\min }=0$ for lenses in the Galactic halo. We use solar mass units, defining $\mu=\frac{M}{M_{\odot}}$, where $M$ is the lens mass. For lenses belonging either to the disc or the bar of the LMC, as well as for the stellar LMC halo contribution, we use the exponential function as in Chabrier (2001) for the mass function $\frac{\mathrm{d} n_{1}}{\mathrm{~d} \mu}$, with integration limits $\mu_{\min }=0.08$ and $\mu_{\max }=1.5$. If the lenses belong to the MW halo or to the LMC halo, the would be MACHOs, we use a set of delta functions with values going from $10^{-5}$ up to $10 M_{\odot}$.

Finally, to compare with the results of the observations, we have to take the expression for the detection efficiency into account, so that we obtain

$\left(\frac{\mathrm{d} \Gamma}{\mathrm{d} T_{\mathrm{E}}}\right)_{\varepsilon}=\frac{\mathrm{d} \Gamma}{\mathrm{d} T_{\mathrm{E}}} \cdot \mathcal{E}\left(T_{\mathrm{E}}\right)$.

To discuss the results of the MACHO collaboration we use the efficiency as a function of $T_{\mathrm{E}}$ as in Alcock et al. (2000b) for which an analytical approximation is given in Paper II. We take the correction reported in Bennett (2005) into account.

\subsection{Expected number and duration}

Following the prescription outlined in the previous section, we evaluate the differential microlensing rate, for each different lens population we consider, along the lines of sight towards the MACHO fields. In particular for each lens population, we calculate the expected number of events per field as

$N_{\text {field }}=E_{\text {field }} \int_{0}^{\infty} \frac{\mathrm{d} \Gamma}{\mathrm{d} T_{\mathrm{E}}} \mathcal{E}\left(T_{\mathrm{E}}\right) \mathrm{d} T_{\mathrm{E}}$

where the "field exposure" $E_{\text {field }}$ is defined in Alcock et al. (2000b), as the product of the number of distinct light curves per field and the relevant time span. Furthermore, we take care to eliminate the field overlaps.

To characterise the expected timescale, we report the median value of the asymmetric distribution $\left(\mathrm{d} \Gamma / \mathrm{d} T_{\mathrm{E}}\right)_{\varepsilon}$, together with the values $T_{\mathrm{E}, 16 \%}$ and $T_{\mathrm{E}, 84 \%}$ that single out the extremes of the $68 \%$ probability range around the median.

In Table 1 we report, for both MW and LMC MACHO lens populations, the total number of the expected events in all the MACHO fields, in the case of a full MACHO halo, together with the expected timescales. We recall that the expected timescale varies with the square root of the mass of the MACHO, and that very short timescales are strongly suppressed by the detection efficiency function.

The predicted durations turn out to be almost independent of the position for both the halo populations we consider, whereas the issue of the variation of the expected timescales with the position across the fields for the self-lensing population was discussed thoroughly in Paper II. Indeed, for lenses in the Galactic halo, we find a dispersion of the median timescales towards the different fields smaller than $1 \%$. In the case of the LMC lenses, the dispersion is only slightly larger, at most $\sim 5 \%$. The expected 
Table 1. Expected duration, median values with $68 \% \mathrm{CL}$ errors, and expected number of events for a full dark matter halo, respectively averaged and summed over the MACHO fields, as a function of the MACHO mass.

\begin{tabular}{ccccc}
\hline \hline Lens mass & \multicolumn{2}{c}{ MW } & \multicolumn{2}{c}{ LMC } \\
$M_{\odot}$ & $T_{\mathrm{E}}$ (days) & $N_{\exp }$ & $T_{\mathrm{E}}($ days $)$ & $N_{\exp }$ \\
\hline $10^{-5}$ & $3.3_{-1.3}^{+3.0}$ & 0.9 & $3.3_{-1.3}^{+3.0}$ & 0.4 \\
$10^{-4}$ & $3.5_{-1.5}^{+3.0}$ & 8.3 & $3.5_{-1.3}^{+3.1}$ & 3.2 \\
$10^{-3}$ & $4.3_{-1.6}^{+3.6}$ & 52 & $5.4_{-1.9}^{+4.4}$ & 13.5 \\
$10^{-2}$ & $8.0_{-3.1}^{+6.5}$ & 115 & $13_{-4.4}^{+8.7}$ & 17.3 \\
0.1 & $20_{-8.0}^{+15}$ & 97 & $36_{-13}^{+21}$ & 9.9 \\
0.2 & $26_{-10}^{+20}$ & 82 & $48_{-17}^{+27}$ & 7.6 \\
0.5 & $41_{-16}^{+29}$ & 59 & $75_{-27}^{+46}$ & 5.0 \\
1 & $55_{-21}^{+38}$ & 44 & $103_{-37}^{+57}$ & 3.4 \\
10 & $149_{-57}^{+79}$ & 12 & $245_{-87}^{+94}$ & 0.5 \\
\hline
\end{tabular}

total number of events due to the LMC stellar halo turns out to be $\sim 0.6$, about half of those due to the LMC disc-bar selflensing contribution (Paper II). The expected median timescale, averaged on the 30 fields, is $T_{\mathrm{E}}=45_{-23}^{+43}$ days.

Overall, we recover the result (Alcock et al. 2000b) that stellar lensing alone cannot explain the signal, so that most of the detected events must belong either to the MW or to the LMC dark matter halo.

\section{The LMC MACHO contribution to microlensing events}

A straightforward conclusion to be drawn from the results on the expected number of events due to the dark matter MW and LMC halos is that, with the implicit hypothesis that the halo fractions in both the MW and the LMC halos are the same, most of the lenses should indeed belong to the Galactic halo. Our aim is to challenge this point of view.

First, we recall the current status about the microlensing events observed by the MACHO collaboration. Next, we carry out a statistical analysis of the observed characteristics of the events (timescale and spatial distribution). The purpose here is to determine to what extent the available data allow the two halo populations to be distinguished. Eventually, using a likelihood analysis based on the microlensing rate, we study whether a viable solution to the MACHO puzzle can come from a significant contribution of a lens population belonging to the LMC halo.

\subsection{The microlensing $M A C H O$ candidates}

In the final analysis of 5.7 years of data in 30 fields towards the $\mathrm{LMC}$, the MACHO group presented two sets of microlensing candidates, sets $\mathrm{A}$ and $\mathrm{B}$, with 13 and 17 candidates, respectively, the former being a subsample of the latter (Alcock et al. 2000b).

Further works allowed more information to be gathered on some of these candidates. The lens for the event LMC-5 is located in the Galactic disc (Alcock et al. 2001b). LMC-22 has been identified as a very likely supernova (Alcock et al. 2001a). LMC-23 has been acknowledged as a probable variable star (Bennett et al. 2005). LMC-9 is a double-lens system with caustic crossing (Alcock et al. 2000a). The microlensing candidates LMC-9, LMC-20, LMC-22, and LMC-27 only belong to set B.

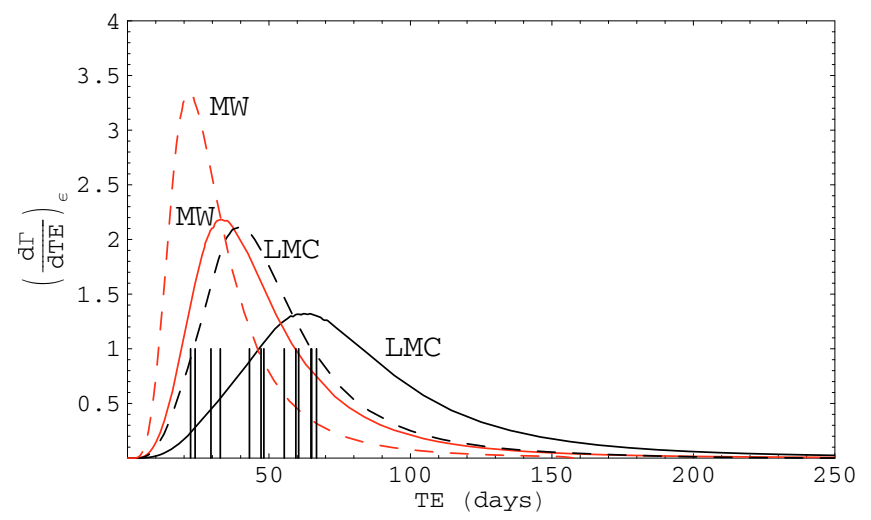

Fig. 2. Normalised differential rate $\left(\mathrm{d} \Gamma / \mathrm{d} T_{\mathrm{E}}\right)_{\varepsilon}$ for both $\mathrm{MW}$ and LMC halos for 0.2 and $0.5 M_{\odot}$, dashed and solid lines, respectively. Superimposed is the value of the observed durations. The $y$-axis values are in $10^{-2}$ units.

For most of the remaining events, a further photometric followup allowed confirmation of the microlensing origin of the flux variation (Bennett et al. 2005).

We restrict the present analysis to a homogeneous set of Paczyński-like events, so we exclude LMC-9. Furthermore, we do not include the Galactic disc lens population, so that we exclude LMC-5, as well as all those candidates whose microlensing origin has been put in doubt. Accordingly, in the following we consider a subset of 13 events taken from the original larger set $\mathrm{B}$, from which we exclude the candidates LMC-5, LMC9, LMC-22, and LMC-23. Furthermore, we have verified that our main conclusions would not change had we started from the smaller set of 11 events, a subsample of the original set A, just excluding the candidates LMC-5 and LMC-23.

\subsection{Duration and position: a statistical analysis}

As previously noted, the expected timescale distributions for microlensing events due to lenses either in the LMC or in the MW halo are almost independent of the position. This property allows us to carry on an analysis in which we compare the observed timescales to the predicted one for each population. In particular, we investigate whether it is possible to draw from such an analysis any conclusion on the relative fraction of the Galactic over the LMC dark matter halo events. Here we neglect the stellar lensing contributions.

For a given value of the MACHO mass, the expected LMC median timescales are larger than the MW ones (Gould 1993). We show in the same plot (Fig. 2) the normalised differential rate distribution for lenses in the LMC and the MW halo, corresponding to two values of the MACHO mass $\left(0.2\right.$ and $\left.0.5 M_{\odot}\right)$. Superimposed, the vertical lines indicate the Einstein time of the observed events. To investigate this issue further, we make use of the Kolmogorov-Smirnov test (hereafter KS). This allows us to test the null hypothesis that the events are drawn from a given population. The resulting KS coefficient gives the significance level of the test. In the first place, we apply the KS hypothesis test separately to the two populations of lenses in the MW and the LMC halo. Then we introduce a parameter $\alpha$, defined as the ratio of the MW events over the total (MW plus LMC events), in order to explore the possibility that an intermediate solution, with the two populations mixed, has to be preferred. To this purpose we look for the value of $\alpha$ that minimises the corresponding KS coefficient. 
Table 2. Kolmogorov-Smirnov test results.

\begin{tabular}{ccccc}
\hline \hline Lens mass $\left(M_{\odot}\right)$ & $k s_{\mathrm{MW}}$ & $k s_{\mathrm{LMC}}$ & $k s_{\alpha}$ & $\alpha$ \\
\hline 0.01 & 1.000 & 1.000 & 1.000 & 0.000 \\
0.10 & 0.999 & 0.683 & 0.683 & 0.000 \\
0.15 & 0.994 & 0.159 & 0.085 & 0.095 \\
0.20 & 0.984 & 0.450 & 0.118 & 0.347 \\
0.22 & 0.969 & 0.655 & 0.144 & 0.471 \\
0.30 & 0.877 & 0.957 & 0.240 & 0.703 \\
0.40 & 0.579 & 0.997 & 0.228 & 0.871 \\
0.50 & 0.176 & 1.000 & 0.176 & 1.000 \\
0.60 & 0.398 & 1.000 & 0.398 & 1.000 \\
0.80 & 0.743 & 1.000 & 0.743 & 1.000 \\
1.00 & 0.911 & 1.000 & 0.911 & 1.000 \\
\hline
\end{tabular}
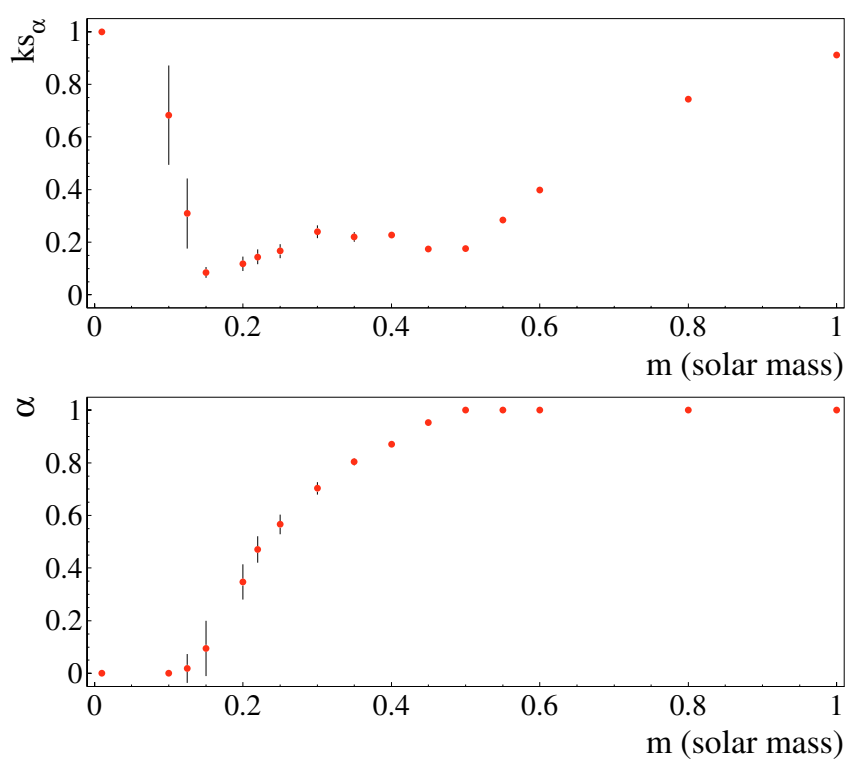

Fig. 3. Kolmogorov-Smirnov and $\alpha$ coefficients as a function of the MACHO mass, with $\alpha$ the ratio of the MW over the total number of dark matter halo events.

To take the variations of the microlensing rate across the fields into account, mainly in the case of lenses in the LMC halo, we carry out the test on each of the MACHO fields separately. We then evaluate and report the value averaged on the 30 MACHO fields. The associated dispersion gives the error bars drawn in Fig. 3. In Table 2 and Fig. 3 we present the results of this analysis. We report the value of the parameter $\alpha$ and the KS coefficient in the three cases considered, $k s_{\mathrm{MW}}, k s_{\mathrm{LMC}}$, and $k s_{\alpha}$, respectively, as a function of the MACHO mass. We report the results only in the range $0.01-1 M_{\odot}$ where the preferred solution are found (see below).

When we consider the MW and the LMC halos separately, the solutions with the highest level of confidence are found for a MACHO mass of $\approx 0.5 M_{\odot}$ and $\approx 0.15 M_{\odot}$, respectively. This result is confirmed by the microlensing-rate-normalised distributions in Fig. 2, where the profile corresponding to the case of $0.5 M_{\odot} \mathrm{MW}$ lenses is almost coincident with that of $0.2 M_{\odot}$ LMC lenses.

For the case of the combined populations, we find that the coefficient $k s_{\alpha}$ presents two minima near the two values of mass found in the previous case. The absolute minimum, which we note is lower than the values obtained in the case of the separate test analysis, is found at $m=0.15 M_{\odot}$ with $\alpha \sim 0.1$, and it gives us the parameters with the highest confidence level. Moreover

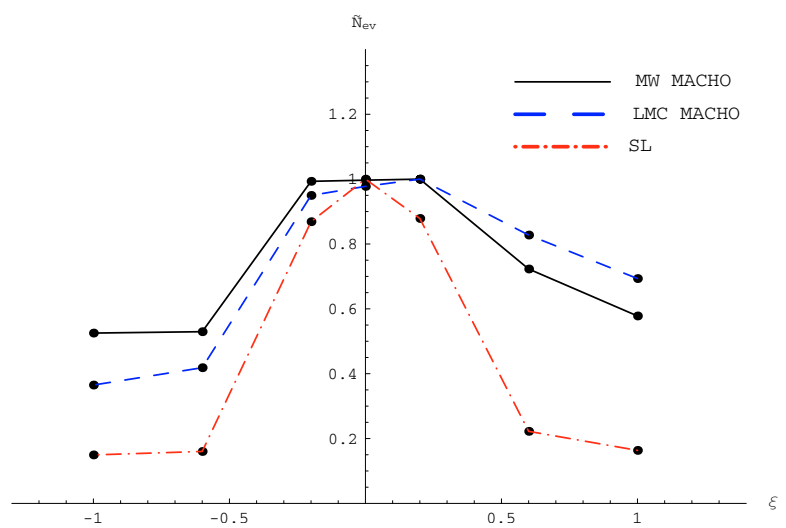

Fig. 4. Normalised expected number profiles along the axis, pointing south-west, orthogonal to the line of nodes and passing through the LMC centre, for different lens populations: self lensing, dot dashed line; MW MACHO, solid line; LMC MACHO, dashed line. Values on the $\xi$ axis are in kpc.

we observe that the $\alpha$ parameter grows monotonically as a function of the mass from 0 up to 1 (Fig. 3).

We conclude that the statistical analysis made on the duration of the events gives a first suggestion that a significant fraction of the observed events could belong to the LMC MACHO halo population.

Next we consider the issue of the spatial distribution of the observed events (Gould 1993). The optical depth profiles clearly show that the LMC halo events are characterised, with respect to LMC self-lensing and to MW halo ones, by a strong asymmetry with respect to the line of nodes (Paper II). Looking at the expected number of events per field, this asymmetry is somewhat weakened but still present, because of the different source density as a function of the position. In order to get insight into the more complex two-dimensional situation we have to deal with, we show the normalised number of the expected events $\left(\tilde{N}_{\text {ev }}\right)$ in Fig. 4, for the different lens populations we consider, evaluated along the axis orthogonal to the line of nodes passing through the LMC centre (the $\xi$ axis in the plot). $\tilde{N}_{\text {ev }}$ has been calculated in the LMC centre and in six more positions, specularly symmetric two by two with respect to the centre. This plot shows clearly that the distribution in the case of self-lensing events is symmetric and, moreover, that outside the bar region it declines sharply. The profile for MW events presents a slight asymmetry with respect to the line of nodes, whereas that corresponding to LMC MACHO lenses have a pronounced asymmetric distribution.

In Paper II we addressed the question of whether the observed asymmetrical distribution of the detected events, which goes indeed in the same sense predicted by a halo LMC population, really does reflect the observational strategy, mainly to argue against the self-lensing origin of the events. Here we take advantage of knowing the expected number of events for all the populations of interest to study this issue in more detail.

We take the line of nodes as the symmetry axis, and as in Paper II we bin the observed space in two regions, the northeast "near" and the south-west "far" ones (Fig. 5). We delimit them by two straight lines parallel to the line of nodes, each at a distance from the latter of $\approx 0.35 \mathrm{kpc}$, the $1 \sigma$ error in position as calculated by van der Marel et al. (2002). Note that this way the innermost LMC regions are excluded from the asymmetry analysis. Our purpose is to test the asymmetry with respect to the prediction of the different lens populations, assigning the probability $p_{i}$ that a microlensing event would fall in the first or second region to be proportional to the expected number of 


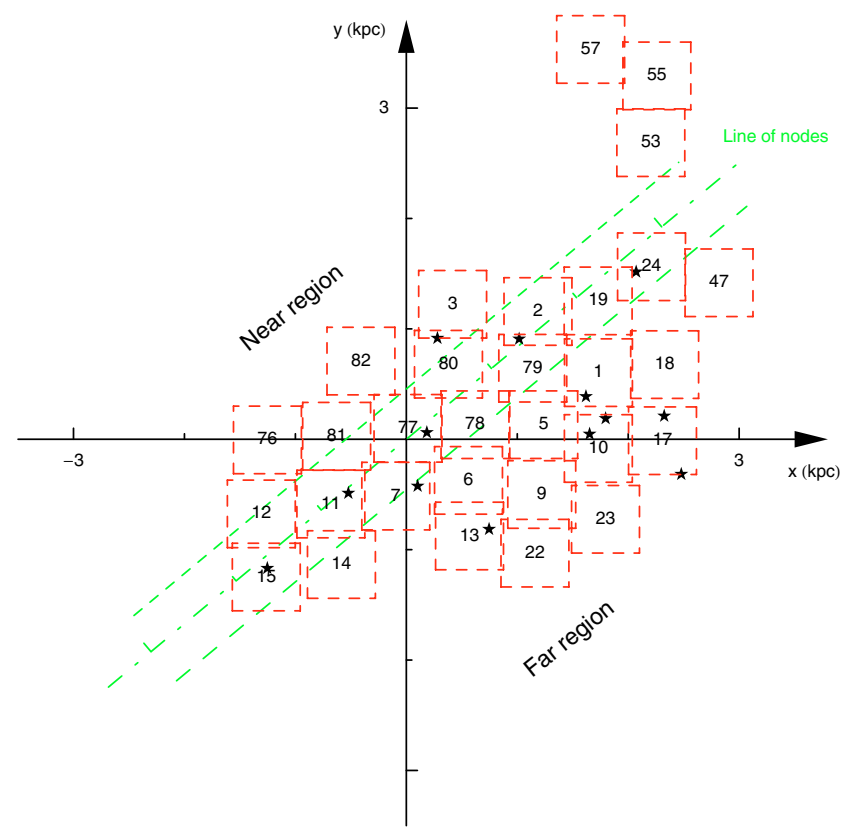

Fig. 5. Location of the $30 \mathrm{MACHO}$ fields in a reference frame centred in the LMC centre with the $x$-axis antiparallel to the right ascension, the $y$-axis parallel to the declination, and the $z$-axis (not shown) pointing towards the observer. The location of the 13 microlensing candidates, a subset of the original set B of MACHO candidates that we use in the present analysis (Sect. 4.1), is shown. Also shown is the position of the line of nodes and the central band around it, which we exclude in the asymmetry analysis.

events of the given population. As in Paper II we make use of the non-parametric Pearson test, which allows us to evaluate the probability to accept the null hypothesis that the $p_{i}$ match the measured distribution, for which we get 1 and 7 observed events in the near and far regions, respectively. This analysis is carried out by normalising the number of expected events of each population to that of the observed events.

The result of this analysis gives us a probability of $46 \%$ and $26 \%$ for a MACHO LMC and MW halo lens population, respectively. This outcome makes the lack of a predominant lens population very clear. Indeed, the expected smoother spatial distribution of MW halo lenses is barely coherent with the observed distribution, thus challenging the explanation that attributes most of the events to this population. Rather, we find much better agreement with the expected asymmetric distribution of LMC halo events. Finally, we note that, contrary to the previous analysis based on the timescale distribution, this result turns out to be independent of the value of the MACHO mass. As a byproduct of the present analysis (confirming that carried out in Paper II), we note that the probability of accepting the hypothesis of a self-lensing origin on the basis of the observed spatial distribution turns out to be only $19 \%$.

Both these analyses, carried out working on normalised distributions (i.e. independent of the actual halo fraction) indicate that a large fraction of the lenses could indeed belong to the LMC dark matter halo.

\subsection{The Galaxy and the LMC: two different halo fractions?}

The previous analysis has provided us with two important clues, both showing that a significant fraction of the events detected by the MACHO collaboration could be part of the LMC dark matter halo. To reconcile this result with the predicted number of events (Table 1), we now drop the hypothesis of equal halo MACHO fractions in the Galactic and the LMC halo. We start by evaluating the likelihood function

$L\left(f_{\mathrm{MW}}, f_{\mathrm{LMC}}\right)=$

$$
\exp \left(-N_{\exp }\right) \prod_{i=1}^{N_{\text {obs }}}\left[E \mathcal{E}\left(T_{\mathrm{E}_{i}}\right) \frac{\mathrm{d} \Gamma}{\mathrm{d} T_{\mathrm{E}}}\left(T_{\mathrm{E}_{i}}\right)\right],
$$

where $f_{\mathrm{MW}}$ and $f_{\mathrm{LMC}}$ are the halo fractions for the MW and the LMC, respectively. For both $N_{\text {exp }}$, the expected number of events, and the differential rate $\frac{\mathrm{d} \Gamma}{\mathrm{d} T_{\mathrm{E}}}$, we sum over all the lens populations (including the stellar ones), multiplying the MACHO contributions for the appropriate halo fraction. The product runs over the $N_{\text {obs }}$ observed events. By Bayesian inversion, using a flat prior probability, it is then possible to compute the probability distribution for the halo fractions given the observed events, $P\left(f_{\mathrm{MW}}, f_{\mathrm{LMC}}\right)$. Note that we are now taking the MACHO mass as a parameter, equal for both halo populations.

In Fig. 6 we show the 2-dimensional probability isocontour for the two halo fractions for four values of the MACHO mass. Eventually, after marginalisation over one variable with respect to the other, we get the results for the two halo fractions as a function of the MACHO mass shown in Fig. 7.

The main outcome of this analysis is that in a significant range of the MACHO mass, the LMC and MW dark matter halo fractions are not expected to be equal. In particular, from 0.1 up to $0.3 M_{\odot}$, both a high value for $f_{\mathrm{LMC}}$ and a low one for $f_{\mathrm{MW}}$ are expected.

This behaviour is mainly due to the observed timescales. It is therefore not surprising that this result is coherent with the one obtained with the KS test, where we found a preferred value of the mass of about $0.2 M_{\odot}$, with a significant expected contribution from LMC MACHO halo lenses. The likelihood analysis gives, for $m=0.2 M_{\odot}, f_{\mathrm{MW}} \sim 4 \%$ and $f_{\mathrm{LMC}} \sim 80 \%$. At face value, given the number of expected events, we get to the overall prediction of about 6-7 events to be attributed to the LMC dark matter halo lens population, 2-3 to the MW halo one, to be looked for among those with the shorter durations, still allowing for a contribution of about 2-3 events, distributed among self-lensing and LMC stellar halo lenses.

For higher values of the mass, the LMC MACHO halo fraction turns out to be almost degenerate though compatible with zero, and, for $0.5 M_{\odot}$, we recover the result of a Galactic MACHO halo fraction of about 20\% (Alcock et al. 2000b). This is again coherent with the issue of the KS test.

We stress that the outcome of this analysis has to be looked at together with the outcomes of the previous analysis on the timescale and the spatial distributions of the observed events. Overall, they indicate that $i$ ) a sizeable fraction of the observed events show characteristics in accord with those expected for a MACHO LMC halo population; ii) such a contribution may be expected by dropping the hypothesis that the halo fractions in the form of MACHOs in the Galactic and the LMC dark matter halo are equal.

As a last point, we discuss the dependence of our results on the choice of the LMC dark matter halo parameters, the central density, and the truncation radius (Sect. 2). We find that any variation in the parameters in a reasonable range around their fiducial values does not change, at least qualitatively, our results. As for the central density, any variation downward (upward) is linearly related to a corresponding change in the total number of expected events. This implies a scaling upward (downward) for the halo fraction without affecting the main conclusion on 

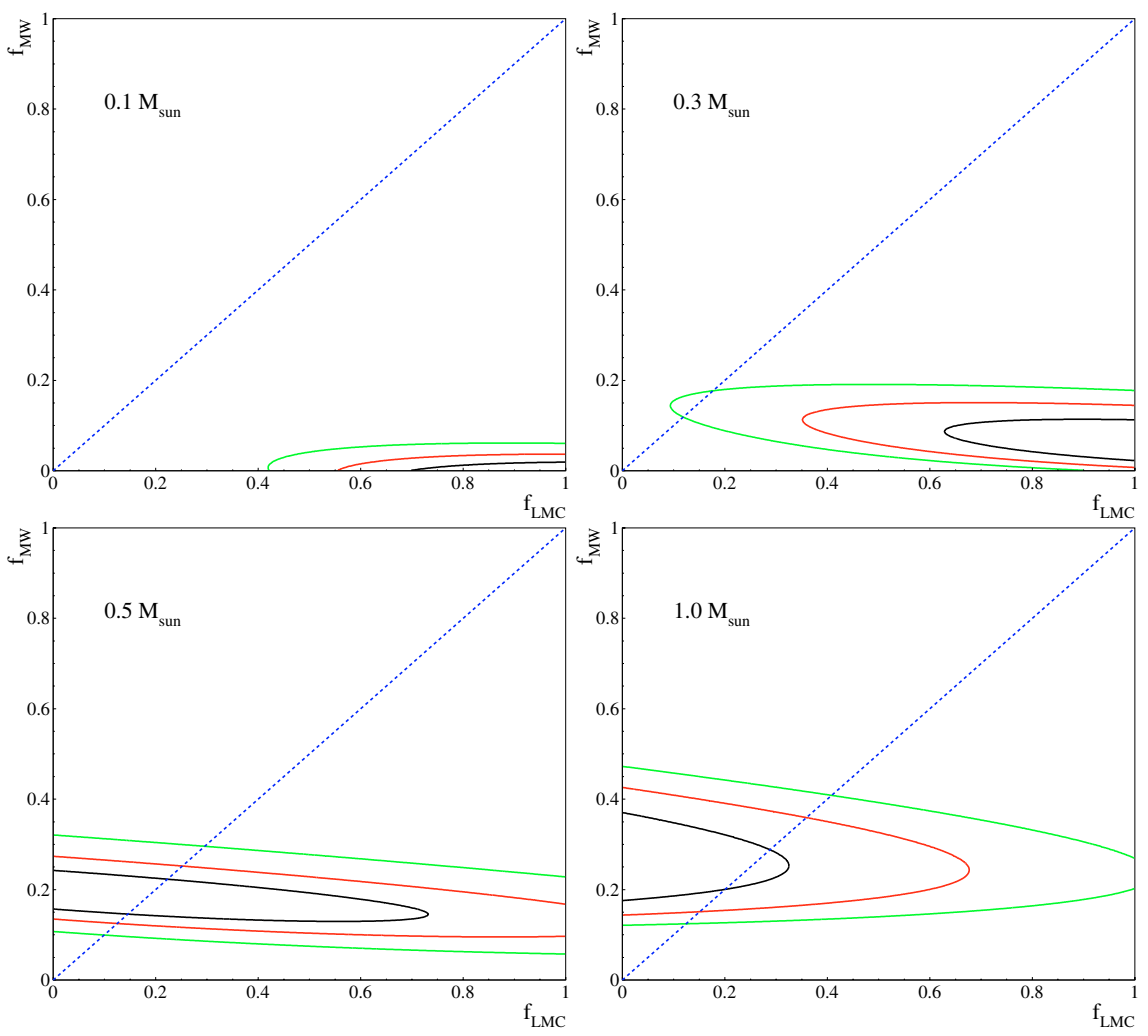

Fig. 6. Probability isocontours with $34 \%, 68 \%$ and $90 \%$ regions for the LMC and MW dark matter halo fraction for four values of the MACHO mass.
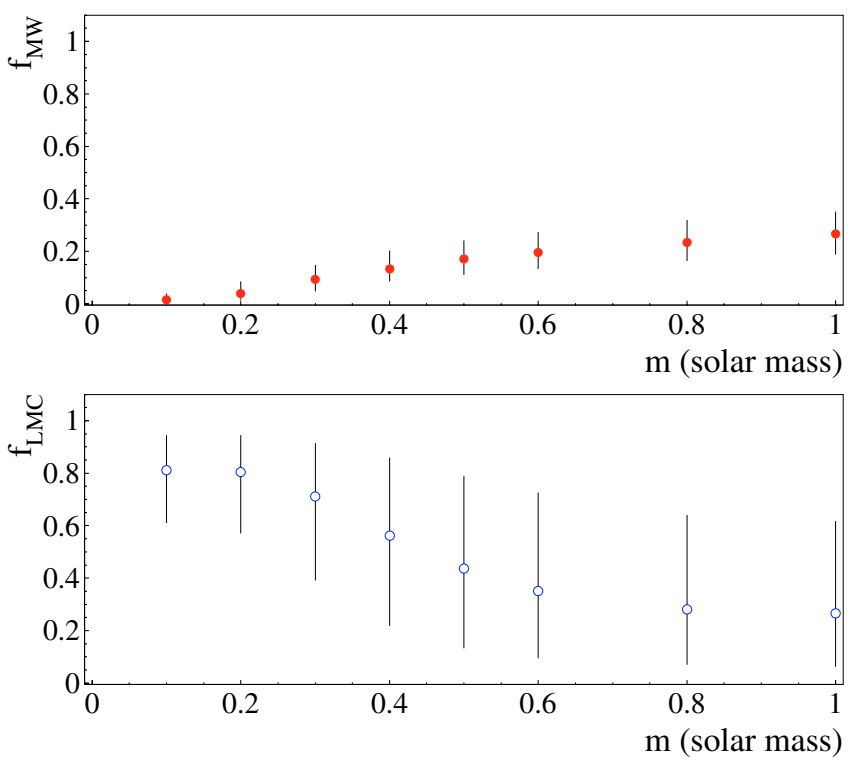

Fig. 7. MW and LMC dark matter halo fraction, median value with $68 \%$ $\mathrm{CL}$ errors, as a function of the MACHO mass.

the contribution of the LMC halo. As for the truncation radius $\left(R_{\mathrm{t}}\right)$, as a first approximation the situation is similar, as a lower (higher) value of $R_{\mathrm{t}}$ roughly give rises to a lower (higher) total LMC mass. However, the issue is slightly subtler. First, the problem is not symmetric with respect to the choice of our fiducial value, $R_{\mathrm{t}}=15 \mathrm{kpc}$. Second, different choices for $R_{\mathrm{t}}$ enhance different spatial distributions for the number of expected events. In particular, $i$ ) the decrease in the number of expected events, for lower values of $R_{\mathrm{t}}$ than the fiducial value, is relatively larger than the increase obtained by choosing higher values; $i$ ) the spatial asymmetry of MACHO LMC halo events is enhanced for a lower value of $R_{\mathrm{t}}$. (Both effects are easily explained, as most of the lenses are expected to be located in the innermost LMC region.) We tested our results with 4 values of $R_{\mathrm{t}}$, respectively lower (higher) of our fiducial value by $20 \%$ and $40 \%$. The relative average decrease in the number of expected events for $R_{\mathrm{t}}=9$ and $12 \mathrm{kpc}$ with respect to the $R_{\mathrm{t}}=15 \mathrm{kpc}$ case is $\sim 20 \%$ and $8.7 \%$, whereas the relative increase for $R_{\mathrm{t}}=18$ and $21 \mathrm{kpc}$ is $\sim 7.4 \%$ and $14 \%$, respectively. As for the spatial distribution, we characterise the asymmetry by the relative difference between the number of expected events evaluated at the extremes of the $\xi$ axis (as defined in Fig. 4) $\xi=1 \mathrm{kpc}$ and $\xi=-1 \mathrm{kpc}$. Where the fiducial case turns out to be of $47 \%$, for $R_{\mathrm{t}}=9$ and $12 \mathrm{kpc}$ we find a relative increase of $11 \%$ and $4 \%$, and for $R_{\mathrm{t}}=18$ and $21 \mathrm{kpc}$ a relative decrease of $2 \%$ and $6 \%$, respectively. Overall, lower values of $R_{\mathrm{t}}$ strengthen our conclusions.

\section{Conclusions}

In this paper we have addressed the issue of the interpretation of the microlensing results toward the LMC. In particular, starting from the sample of microlensing candidates reported by the MACHO collaboration, we discussed the contingent contribution of a lens component belonging to the LMC dark matter halo besides that of the Galactic one. As a main result of the present analysis, we show that a sizeable fraction of the observed events, up to about half of the total, could indeed be part of the first component.

We summarise our analysis as follows. First, we compared the observed timescales with those expected for the two different MACHO populations, the MW and the LMC. As a result, we have shown that the preferred values for the MACHO mass are about 0.5 and $0.2 M_{\odot}$, respectively and, through a KS test, that the latter solution is preferred. Second, we studied the spatial distribution of the observed events, recalling that, because of the inclination of the LMC disc with respect to our line of sight, an asymmetry is expected for LMC halo events. As a result we have 
shown that, independent of the value of the MACHO mass, the observed distribution better matches that expected for a LMC halo population than for a MW halo population. Overall, these are clues suggesting the presence of a significant MACHO LMC halo population among the observed events.

The extremely high value of the overall Galactic halo mass compared to the LMC one implies that generally one can safely ignore the LMC halo component. To explain such a large contribution of the latter, one way out is to consider that the halo fractions in the form of MACHOs of the two components, the MW, and the LMC halos may be different. Coherently with the timescale analysis, this issue turns out to be strongly dependent on the value of the MACHO mass.

In order to get to more quantitative results, we tested this hypothesis through a likelihood analysis. Eventually we have shown that for a wide range of mass values a different (and larger) fraction for the LMC halo compared to the MW one is indeed expected. In particular, for a MACHO mass of $\sim 0.2 M_{\odot}$, we evaluated a high halo fraction for the LMC, $\sim 80 \%$, together with a small one for the MW, $\$ 5 \%$, thus implying that about half of the observed events should belong to the LMC dark matter halo. On the other hand, for MACHOs of $\sim 0.5 M_{\odot}$, we recovered the well-known result of a MW halo fraction $\sim 20 \%$ with a (possibly) negligible contribution from the LMC dark matter halo.

A possible explanation of the origin of different halo fractions could come from the different formation histories of the two galaxies, or more simply, could be related to the fact that one observes all the LMC halo but, practically, only a line of sight through the Galactic halo.

These conclusions should be taken cum grano salis. The overall implicit assumption is the validity of the MACHO results, whereas they are actually challenged by the EROS collaboration. Furthermore, an intrinsic limit for all the analyses carried out up to now is the result of the lack of available statistics. The SuperMACHO collaboration (Becker et al. 2004) is expected to provide a larger sample of candidates spread over a much larger field of view, and this should allow the problem to be constrained further. Eventually we stress that the model issue, in particular for the LMC components, is still a matter of debate. Our analysis shows, however, that it is in principle possible to characterise and distinguish the two halo lens populations and, moreover, challenge the usual implicit assumption of equal halo fractions in the form of MACHO for both the Galactic and the LMC dark matter halos.

Acknowledgements. We thank Andy Gould for useful comments and discussions. S.C.N. was partly supported by the Swiss National Science Foundation. F.D.L. work was performed under the auspices of the EU, which has provided financial support to the "Dottorato di Ricerca Internazionale in Fisica della Gravitazione ed Astrofisica" of Salerno University, through "Fondo Sociale Europeo, Misura III.4". G.S. acknowledges support for this work provided by MIUR through PRIN 2004 "Astroparticle Physics", protocol number 2004024710_006, and by research funds of Salerno University.

\section{References}

Afonso, C., Albert, J. N., Andersen, J., et al. 2003, A\&A, 400, 951 Alcock, C., Allsman, R. A., Alves, D., et al. 2000a, ApJ, 541, 270 Alcock, C., Allsman, R. A., Alves, D. R., et al. 2000b, ApJ, 542, 281 Alcock, C., Allsman, R. A., Alves, D. R., et al. 2001a, ApJ, 552, 582 Alcock, C., Allsman, R. A., Alves, D. R., et al. 2001b, Nature, 414, 617 Alves, D. R. 2004, ApJ, 601, L151

Ansari, R., Aurière, M., Baillon, P., et al. 1997, A\&A, 324, 843 Becker, A. C., Rest, A., Stubbs, C., et al. 2004 [arXiv: astro-ph/0409167] Bennett, D. P. 2005, ApJ, 633, 906

Bennett, D. P., Becker, A. C., \& Tomaney, A. 2005, ApJ, 631, 301 Calchi Novati, S., Paulin-Henriksson, S., An, J., et al. 2005, A\&A, 443, 911 Chabrier, G. 2001, ApJ, 554, 1274

Cole, A. A., Tolstoy, E., Gallagher, J. S., \& Smecker-Hane, T. A. 2005, AJ, 129, 1465

de Jong, J. T. A., Widrow, L. M., Cseresnjes, P., et al. 2006, A\&A, 446, 855 de Rujula, A., Jetzer, P., \& Masso, E. 1991, MNRAS, 250, 348

Gallart, C., Stetson, P. B., Hardy, E., Pont, F., \& Zinn, R. 2004, ApJ, 614, L109 Gerhard, O. E. 1993, MNRAS, 265, 213

Gould, A. 1993, ApJ, 404, 451

Green, A. M., \& Jedamzik, K. 2002, A\&A, 395, 31

Griest, K. 1991, ApJ, 366, 412

Gyuk, G., Dalal, N., \& Griest, K. 2000, ApJ, 535, 90

Jetzer, P., Mancini, L., \& Scarpetta, G. 2002, A\&A, 393, 129, Paper I

Lasserre, T., Afonso, C., Albert, J. N., et al. 2000, A\&A, 355, L39

Mancini, L., Calchi Novati, S., Jetzer, P., \& Scarpetta, G. 2004, A\&A, 427, 61 (Paper II)

Minniti, D., Borissova, J., Rejkuba, M., et al. 2003, Science, 301, 1508

Paczyński, B. 1986, ApJ, 304, 1

Roulet, E., \& Mollerach, S. 1997, Phys. Rep., 279, 67

Tisserand, P., \& Milsztajn, A. 2005 [arXiv:astro-ph/0501584]

van der Marel, R. P. 2004 [arXiv: astro-ph/0404192]

van der Marel, R. P., Alves, D. R., Hardy, E., \& Suntzeff, N. B. 2002, AJ, 124, 2639

van der Marel, R. P., \& Franx, M. 1993, ApJ, 407, 525 